\title{
Progresses in Fetal Monitoring by Improved Data Acquisition
}

\author{
Joachim H. Nagel \\ University Erlangen-Nurnberg, Germany
}

$S^{\prime \prime}$ NCE the possibilities of gaining information on the fetal condition by non-invasive means are limited to only a few procedures, such as recording of fetal heart action and ultrasonic imaging, expanding the diagnostic potential is a great biomedical engineering challenge. Instead of searching for new, complicated measuring techniques that are often declined by the medical staff, this challenge can be met by developing suitable procedures aimed at improving the analysis of data provided by present routine examinations. Cardiotocography (CTG) is the most commonly used method in perinatal monitoring, but serious difficulties still attach to its interpretation. In many cases, an unequivocal diagnosis is not possible without first obtaining additional physiological information. The reasons may be either physiological or due to shortcomings of existing instrumentation. Both problems could be solved by improving the processing of available bioelectric signals, and by developing a new physiological model of the maternal influence on the fetal cardiovascular system, permitting a more reliable interpretation of the CTG.

\section{Fetal Heart Rate Recording}

Heart action is the only vital function of the fetus that can be recorded effectively without lesion. Consequently, fetal heart rate (FHR) analysis, related to uterine pressure, is the most important method in prenatal monitoring. Using non-invasive methods, heart action can be assessed by:

- the electrocardiogram (ECG), picked up from the material abdomen,

Dr. Joachim H. Nagel is Akademischer Rat at the Central Institute for BioMedical Engineering of the University Erlangen-Nürnberg, Germany. He received the diploma in Physics from the University of Saarbrücken, Germany in 1973, and the Ph.D. in Natural Sciences from the University Erlangen-Nürnberg, Germany in 1979.
- the Doppler-shifted ultrasonic heart echo, and

- the fetal heart sounds, the phonocardiogram.

Of these three methods, the ECG is the most suitable for accurate beatto-beat determination of the fetal heart rate, since it is not only a measure of the movement of individual parts of the heart, like the phonocardiogram and the ultrasonic heart echo, but it is indeed the stimulus for contraction. However, the external measurement of the ECG is complicated by interference from other signals, such as the maternal ECG and abdominal muscle action potentials (EMG), and by significant attenuation of the ECG by the tissues between the heart and the electrodes. The poor signal-to-noise ratio and the similar frequency spectra of the signal and noise components make an analysis of the ECG using conventional filtering techniques rarely possible.

The two main problems of ECG analysis are elimination of the maternal electrocardiogram (MECG) and localisation of the fetal electrocardiogram (FECG) QRS complexes within the remaining disturbances. It is common practice to separate maternal and fetal ECG's by using some form of amplitude discrimination, taking advantage of the much greater amplitude of the MECG. The abdominal signal is filtered in such a way that the ECG's are reduced to only the QRS complexes. There are two triggering levels, one for the detection of the maternal QRS complexes and a lower one for localising those of the fetus. The disadvantage of this method is obvious; each time a maternal QRS complex is detected the signal is completely suppressed, and coincident fetal complexes-often $50 \%$ and more-are lost. Moreover, there is no easy way of deciding whether the lower triggering level has been crossed by the fetal ECG or by signal disturbances. Thus, because of the poor signal-to-noise ratio and the high rate of coincidence between maternal and fetal ECG's, the detection rate for the fetal QRS complexes is no better, on the average, than about $60 \%$. Consequently, it is evident that a reliable FECG analysis first requires the MECG to be subtracted in such a way that the remaining FECG is not affected. The resulting fetal QRS complexes may then be detected by means of a correlation analysis which is able to distinguish between them and the interfering noise ${ }^{[1]}$.

The MECG is additively superimposed upon the FECG, and multiplicative distortions that result mainly from movements can be disregarded. Theoretically, it is therefor possible to separate the MECG, if its waveform is known, from the abdominal signal by subtraction. In practice, it is rather difficult to perform the subtraction, since the waveform of the MECG is not known in advance and is often subject to fluctuations within the time interval in which the signals are being processed. Other groups tried to solve this problem by means of an additional MECG lead; the elimination of the MECG from the abdominal signal then being achieved by subtracting the two ECG's. This method is fraught with problems since it is practically impossible to bring the two maternal ECG's into amplitude and phase coincidence. A much better method is the use of an averaging technique to extract the waveform of the MECG, using only the abdominal lead. Because of their large amplitude in the abdominal signal, the R-waves of the MECG are easily detectable by means of a threshold detector. With a running average of succeeding intervals of the abdominal signal, all containing the maternal QRS complex in the same phase position, a reference signal corresponding to one interval of the MECG is obtained ${ }^{[2,3]}$. The FECG and the EMG are suppressed in the reference since they are statistically independent of the MECG. Subtraction of the reference from the abdominal signal then results in complete elimination of the MECG. Amplitude variations of the MECG which would result in nonzero differences, require the reference signal to be scaled to the actual MECG amplitude before each subtraction. A running average guarantees the adaptation of the reference signal to varying waveforms of the MECG. It is thus possible to detect all the fetal QRS complexes in spite of their coincidence with the MECG. Figure 1 shows an example of the sub- 


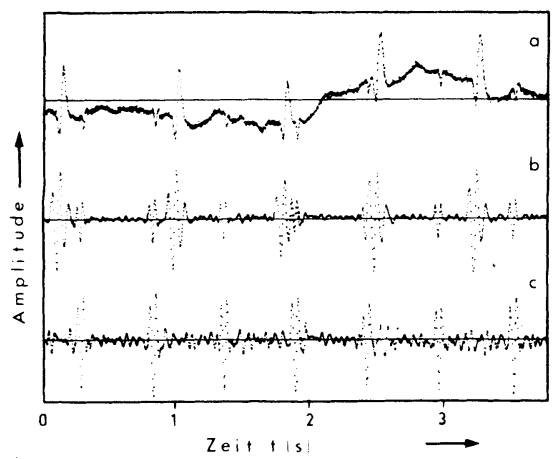

Figure 1. Subtraction of the maternal ECG from the abdominally measured signal. (a) original abdominal ECG (b) filtered ECG (c) MECG subtracted.

traction of the MECG, the average attenuation is about $30 \mathrm{~dB}$. For exact subtraction of the MECG, it is necessary that both phase and amplitude be known for the template and the actal R-wave. This condition cannot be satisfied in all cases by simple threshold logic. The superposition of the FECG and the EMG sometimes leads to jitter in the R-wave detection, and consequently to a faulty determination of amplitude and phase of the actual maternal R-wave. In order to prevent these errors, the MECG is analyzed using a cross-correlation algorithm. The maximum of the cross-correlation function between abdominal signal and maternal ECG template localises the MECG R-wave with greater precision than does the simple threshold detector ${ }^{[4]}$. The scaling factor needed for matching the template and the actual MECG is the quotient of the cross-correlation and the auto-correlation template functions, with zero delay time for both. Using specially developed algorithms, computation is reduced to a minimum, and even slow microcomputers are capable of performing the subtraction in real-time. ${ }^{[1]}$

The remaining signal disturbances, which are caused mainly by the maternal EMG, prevent the fetal ORS complexes from being detected with the necessary accuracy using simple threshold logic. Because of the poor signal-to-noise ratio, the expected error using threshold detection of the FECG is, on average, greater than $25 \%$, including both false positive and false negative detection (provided that the MECG has been subtracted without eliminating coinciding fetal $R$ peaks). A reference of the FECG is extracted from the abdominal signal by coherent averaging, as in processing of the MECG. The cross-correlation function between the reference and the abdominal signals is computed with an integration time of slightly less than one FECG interval. This interval ensures an adequate increase in the signal-to-noise ratio only because of prior subtraction of the MECG ${ }^{[1,5]}$. Using integration times greater than one signal cycle would prevent the detection of one single $R$ peak, and it would be possible to gain knowledge only about the mean interval between succeeding R-peaks ${ }^{[6]}$. The maxima of the cross-correlation function mark accurately the position of the fetal QRS complexes. The two main advantages of cross-correlation analysis are that:

-the fetal electrocardiogram is detected even if its amplitude is smaller than that of the superimposed noise and,

- the jitter of the trigger point on the $R$-wave due to signal disturbances, such as appears when performing simple threshold detection, is substantially reduced.

The performance of this method has been assessed in clinical trials. Comparing the results with those obtained using the earlier techniques of abdominal ECG processing showed a mean increase in the detection rate of the fetal QRS complexes of about $60 \%$. When compared with fetal heart rate recordings using ultrasonocardiography or phonocardiography, the new method shows an important improvement with respect to the evaluation of short term variations of heart rate.

\section{Recording of Uterine Activity From the Abdominal Lead EMG}

Monitoring uterine activity is important in prenatal medicine for surveying the course of the pregnancy and evaluating the condition of the fetus. Two methods, one internal and the other external, are known for recording uterine activity. With both techniques the activity is evaluated in terms of uterine pressure, the tocogram. For internal tocography, the pressure is determined by introducing a catheter into the uterine cavity. It is possible, with this method, to determine the absolute intrauterine pressure. Often, however, internal tocography is rejected because of the practical difficulties involved. Moreover, the use of a catheter may influence parturition, and so it should not be used in premature deliveries, where monitoring of the contraction activity would be of particular significance. With external tocography, some form of pressure or force transducer is fastened to the abdominal wall by means of an elastic belt.
Uterine contractions produce changes in the displacement of a sensor pin which acts on the transducer, producing an electrical signal that corresponds to the relative uterine pressure. External tocography, however, has the drawback that it is subject to many factors which may influence the results of the measurement. I feel that the limtations of tocography cannot be eliminated by technical improvements of the measuring equipment. Recording the uterine contractions from the abdominal lead electromyogram (EMG), as an alternative to conventional tocography, has, therefor, been studied ${ }^{[7]}$.

Changes in intrauterine pressure are produced by contractions of the uterine muscle. Contraction is stimulated by a series of action potentials. The force developed by the muscle depends on the number of activated, i.e., depolarising fibers within the muscle, and on the frequency of stimulation of each individual fiber ${ }^{[8-12]}$. The time constant of the contraction is large compared to that of the stimulating pulse, and the resulting force, $f(t)$, can be written as:

$$
f(t)=c_{1} \cdot \sum_{n=1}^{N} s_{n}(t)
$$

where $c_{1}$ is a constant, $N$ is the total number of fibers in the muscle, and $s_{n}(t)$ is the firing rate of the $n$-th fiber. The linear summation is not completely valid because of non-linear properties of the muscle fibers. However, it is a good first approximation and is sufficient to describe the basic relationships. The change of intrauterine pressure that results from the force of the uterine muscle during contractions can be approximated using the simple model of a fluid-filled balloon. Pressure is propagating equally in all directions, and muscular force is directed tangentially to the surface of the balloon. It is well known that the change of the internal pressure of the balloon is proportional to the external force, i.e.

$$
\Delta p(t)=c_{2} \cdot f(t)
$$

where $c_{2}$ is a constant. The absolute intrauterine pressure can be written as:

$$
\begin{gathered}
p(t)=p_{0}+c_{2} f(t) \\
=p_{0}+c_{3} \cdot \sum_{n=1}^{N} s_{n}(t)
\end{gathered}
$$

where $p_{0}$ represents the base-line pressure. When a muscle fiber depolarises, an action potential is pro- 
duced which travels along the fibre. The shape of the action potential depends on the fiber size, the propagation velocity, and the geometric relationship of measuring electrode to fiber. By applying electromagnetic field theory, the electrode potential can be calculated for any given geometry. The biphasic action potentials of individual muscle fibers are summed at a surface electrode, and the recorded potential is the electromyogram. As the stimulation of different fibers is not synchronised, a complicated interference pattern is observed which is very sensitive to the firing rate and pattern of the individual $\mathrm{fi}-$ bers and to the position of the electrode ${ }^{[13,14]}$. Rectifying and low pass filtering of the EMG produces a measure of its intensity (IEMG). The IEMG measured from a single electrode can be estimated as:

$\operatorname{IEMG}(t)$

$$
=c_{4} \sum_{n=1}^{N} 1 / r_{n}^{2} \cdot \cos \Phi_{n} \cdot s_{n}(t)
$$

where $c_{4}$ is a constant, $s_{n}$ again denotes the firing rate of the $n$-th fiber, $N$ is the total number of fibers, $r_{n}$ is the distance between the $n$-th fiber and the electrode, and $\Phi_{n}$ is the angle between the $n$-th fiber and the connecting line to the electrode. This equation for the IEMG assumes that the muscle fiber has a straight cylindrical shape, that its length is small compared with the distance to the electrode, and that the conductivity of the body is homogenous. Without these assumptions, the computation becomes more difficult, but the result would be essentially the same. Another simplification is that the superposition of the biphasic action potentials of the individual muscle fibers results in a linear dependency of the intensity of the EMG on the number of activated fibers. This simplification is not really valid for all muscles, as superposition is influenced by the probability distribution for the pattern of stimulation of the single fibers. In the special case of the uterine muscle, this assumption is in accordance with the results of experimental studies. Comparing equations (2) and (3), it becomes evident that there is a high correlation between intrauterine pressure and IEMG. The major difference is in the dependency of the IEMG on electrode position. If the distance $r_{n}$ and the angle $\Phi_{n}$ were equal for all fibers, the intrauterine pressure changes would be proportional to the IEMG. Unfortunately, this condition cannot be fulfilled because of the great extent of the uterine muscle,

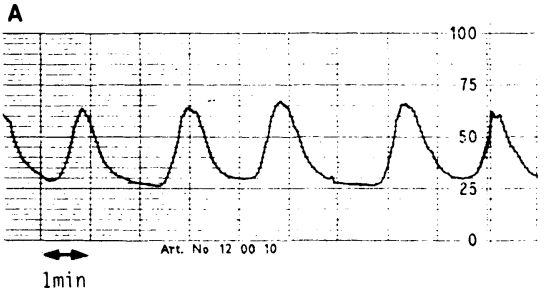

uterine muscle or the propagation of the contractions can be monitored. The waveshape of the IEMG is strongly dependent upon the time constant of the lowpass filter. Using a small time constant $(<1 \mathrm{sec})$ results in a very ragged waveform. Greater time constants involve a smoother waveform, at the expense of some loss of transient response.

The abdominal lead EMG of the uterine muscle is disturbed by the maternal ECG, the fetal ECG, and the abdominal muscle EMG. The maternal and fetal ECG's can be eliminated by either using appropriate bandpass filters $(150-250 \mathrm{~Hz})$, or by using the subtraction algorithm described previously. As the contractions of the abdominal muscle also result in intrauterine pressure changes, it is not necessary to eliminate its EMG from the abdominally derived signal. Figures 2 and 3 show a comparison of uterine activity curves obtained from an external pressure transducer and from the EMG. The EMG has been measured using two electrodes, attached to the isthmus uteri and the fundus uteri. Figure 2 shows good correspondence between the externally measured pressure and the uterine activity recorded from the IEMG. However, it can be seen that the IEMG contains more information about the progressive increase in intensity of the contractions as a reaction of the uterine muscle to an oxytocin infusion. When analysing the cardiotocogram from
Using other electrode configurations, the activity of particular parts of the

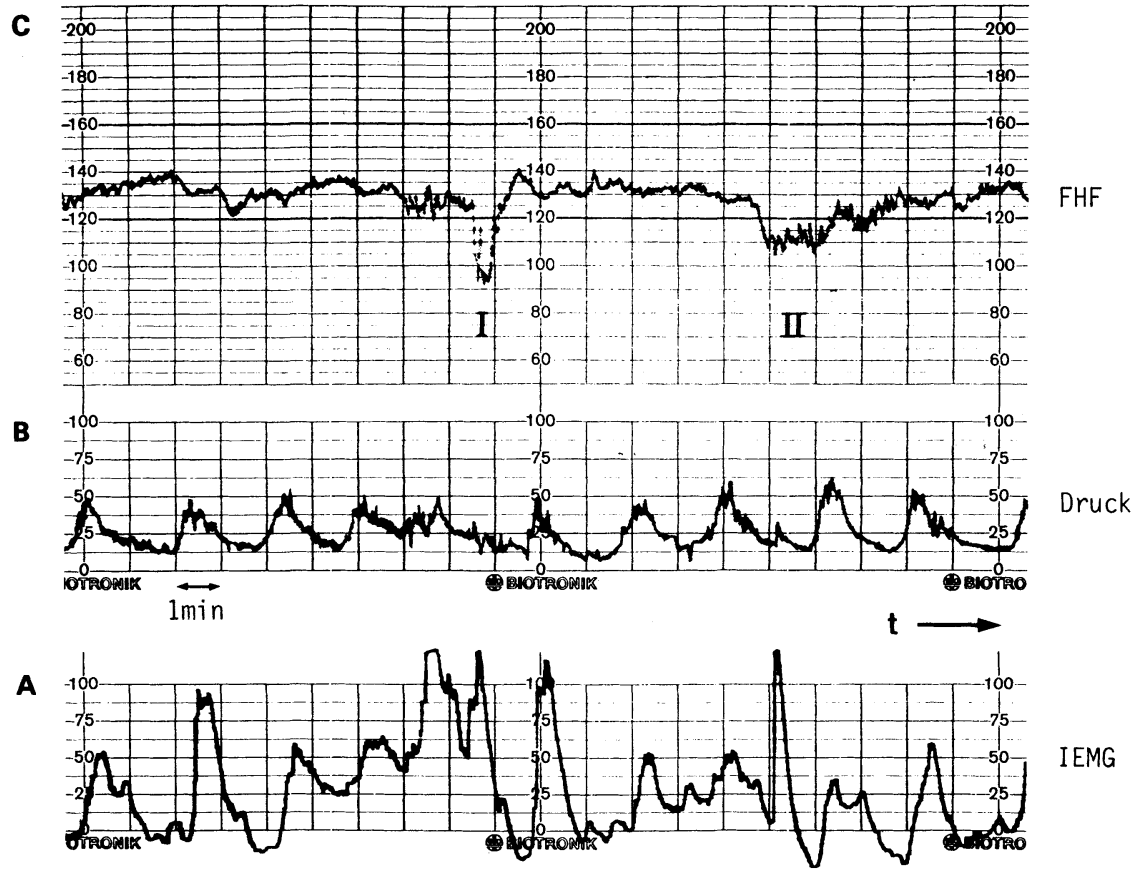

Figure 3. Cardiotocogram. The FHR is computed from the scalp lead ECG (C), the uterine pressure is determined by an external pressure transducer (B) and from the IEMG (A). 
Figure 3, it is evident that the correlation between FHR and IEMG is greater than between FHR and externally measured pressure.

Using a single set of abdominally attached electrodes provides the means of obtaining extensive data, while giving the minimum discomfort to the patient and enabling the physician to obtain an accurate picture of the state of both mother and fetus.

\section{The Correlation of MHR and FHR-An Important Aspect of CTG Interruption}

There is no question that cardiotocography plays an extremely important role in prenatal monitoring. It is thus all the more surprising that a standardised scoring system has still not been developed for the evaluation of the CTG. Accordingly, in everyday practice uncertainties still exist with respect to the analysis of the CTG and, unfortunately, misinterpretations still occur. Critics even claim that too much importance is frequently attached to the CTG, with the result that unnecessary caesarian sections are performed. The question arises whether the existing models of the origin of FHR fluctuations are comprehensive. Conversely, shortcomings of measuring equipment presently available can result in the non-detection of situations hazardous for the fetus.

When comparing simultaneous recordings of FHR and MHR, a considerable similarity of the tracings becomes apparent (Fig. 4). The question arises as to whether the correlation between FHR and MHR represents a random event. I feel sure that there is a systematic dependence. Fetal oxygen availability is a direct function of maternal cardiac output and oxygen saturation, as well as of placental sufficiency. It is well known that a reduction in available oxygen usually influences the fetal heart rate through the action of the cardiac regulatory system. An oxygen deficit is compensated by acceleration of the heart rate to ensure an adequate supply to the fetal tissues. As a consequence, not only placental insufficiency, but also a change in maternal blood supply should find its expression in the FHR. So far this is nothing new. What is important, however, is to draw the right conclusions. CTG interpretation usually correlates FHR and uterine pressure. But what is to be done in the absence of pressure changes, or if an abnormal pattern presents during labour? Can we be sure that the increasing uterine pressure is the reason for heart rate variations in every case? And what influence has the maternal cardiovascular system? A consideration of the physiological situation leads to surprising results. Labour activity represents a stress situation, not only for the fetus but also for the mother, and results in maternal heart rate variations and intensified respiration. Some authors have already demonstrated an augmented $\mathrm{pO}_{2}$ during labour. With normal maternal cardiac output and placental sufficiency this produces an increase in the fetal oxygen supply. The physiological reaction will be a deceleration of fetal heart rate, or DIP, as is indeed observed. On the other hand, fetal stress caused by uterine contractions would, as a normal physiological reaction, result in an acceleration comparable to that of the maternal heart. Obviously, there are two opposing regulatory processes. So should not the commonly observed decelerations logically be attributed to the maternal cardiovascular system rather than to uterine pressure? An obvious case is shown in Figure 4. Normal reaction to labour activity is an acceleration of MHR and a fetal DIP. The first contraction on the left-hand side, however, shows no maternal response and thus no deceleration of FHR. On the other hand, FHR accelerations and decelerations can often be seen that are not interpretable as being due to uterine pressure or fetal movements. They, too, find their logical explanation in MHR variations.

A second point of interest is the interpretation of the onset of the DIP's. If the previous statement holds, then these DIP's must also be related to the changed conditions of the maternal cardiovascular system. Again, the consequences are quite surprising. With the onset of a uterine contraction, a maternal reaction is first detected as a change in heart rate. The associated increase in $p \mathrm{O}_{2}$ results in fetal heart rate deceleration. But there must be a delay vis-a-vis the acceleration of the maternal heart, since the passage of oxygen through the placenta takes a certain time. The delay time itself will depend upon placental efficiency. In common with umbilical cord compression, placental insufficiency retards the increase in fetal $\mathrm{pO}_{2}$ and thus heart rate deceleration. The CTG thus shows a late deceleration. But the essential point is that late decelerations do not automatically mean placental insufficiency or any other disorder, since a second time constant that may be responsible must be taken into account; namely, the time delay in the maternal response to uterine contraction which may be increased without any
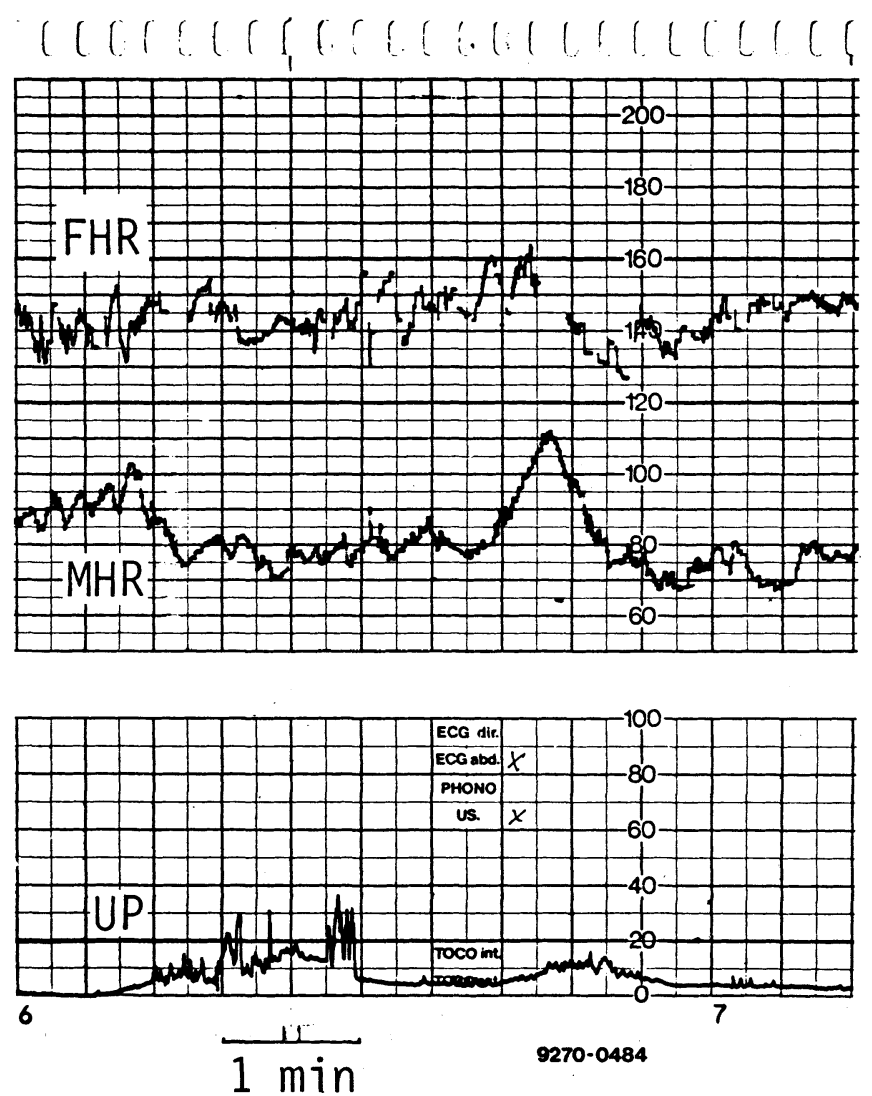

Figure 4. Simultaneous recording of CTG and MHR showing the predominant influence of MHR on fetal heart rate. 
consequences for fetal well-being. This accords with the experience of other authors who found, without knowing the physiological reason, that a late DIP should not be interpreted as a sign of risk, provided the FHR oscillations remain within their normal range. Therefore, it is obvious that the unequivocal interpretation of DIP's would be considerably facilitated by a knowledge of the maternal condition. Figure 4 shows the simultaneous recording of both maternal and fetal heart rates, together with uterine pressure. There is a remarkable conformity of FHR and MHR, but with inverted sign of the fluctuations.

Analysis of the correlation between FHR and MHR provides important information. The correlation coefficient is largest when the maternal heart rate recording is delayed by 30 to 40 seconds vis-a-vis the FHR curve. This delay is the response time of the placenta and fetal regulatory system. In other words, if the maternal oxygen supply changes, it will take about 30 seconds for the FHR to respond. This is an important sign, which permits an assessment of placental sufficiency by following the time delay. Insufficiency will increase the delay and reduce the correlation. Since the response time is affected essentially by the reserve capacity of the placenta, the latter can also be judged.

Heart rate is, of course, a rather poor indicator of physiological condition for both the fetus and the mother.
Even such simple measurements as maternal blood pressure can provide additional information. However, for an accurate analysis of the feto-maternal coupling, at least cardiac output and maternal oxygen saturation should be monitored. Nevertheless, extensive investigations have shown that, for routine use, even the MHR provides sufficient information to improve the interpretation of the CTG quite considerably. The correlation analysis of fetal and maternal heart rates facilitates diagnose, particularly in borderline cases, and may help to prevent misinterpretation of the CTG.

\section{Conclusions}

The results of the studies discussed here show that the processing and interpreting of routinely acquired fetal and maternal data has, so far, not been fully utilized. It would thus appear to make good sense, not only to continue the search for new measuring methods for determining additional physiological parameters, but also to pursue attempts to improve the evaluation of presently obtainable data.

All in all, the procedures described have proved valuable in clinical trials. The simplicity of simultaneous recording of fetal and maternal heart activity in addition to uterine contraction is highly suitable for routine clinical work. The proposed extension of CTG interpretation by including the maternal cardiovascular condition will pro- vide improved surveillance of both mother and fetus during pregnancy and parturition.

\section{References}

1. Nagel J: Analyse bioelektrischer Potentiale in der Perinatologie. Dissertation Universität Erlangen, 1979

2. Rhyne VT: Medical Research Engineering, 22. August-September, 1969.

3. Rhyne VT: IEEE Trans. on Bio-Medical Engineering, Vol. BME-16, No. 1, 80, 1969.

4. Rex RL, Roberts GT: Hewlett-Packard Journal, Vol. 21, No. 3, 1969.

5. Bendat JS: Principles and applications of random noise theory. Wiley, ch. 7, New York, 1966.

6. Bemmel van JH: Detection and processing of foetal electrocardiograms, Institute of Medical Physics TNO, Utrecht, 1969.

7. Nagel J, Schaldach M: The non-invasive assessment of uterine activity. In: Rolfe $P(E d)$ : Non-invasive Measurements vol 2, Academic Press Inc. (London) Ltd. pp 103-129, 1983.

8. Calvert TW, Chapman AE: Proceedings of the IEEE, Vol 65, No. 5, 682, 1977.

9. De Luca CJ, Forrest WJ: Some properties of motor unit action potential trains recorded during constant force isometric contractions in man. Kybernetik 12, 160-168, 1973.

10. Dill LV, Maiden RM: The electrical potentials of the human uterus in labor. Am. J. Obstet. Gynecol. 52, 735, 1946.

11. Lindström LH, Magnusson I: Interpretation of myoelectric power spectra: a model and its applications. Proceedings of the IEEE, Vol. 65 No. 5, 653, 1977 .

12. Wolfs GM, van Leeuwen M: Electromyographic observations on the human uterus during labour. Acta Obstet. Gynecol. Scand. Suppl. 90.

13. Clamann HP: Statistical analysis of motor unit firing patterns in a human skeletal muscle. Biophys. J. 9, 1233-1251, 1969.

14. Milner-Brown HS, Stein RB: The relation between the surface electromyogram and muscular force. J. Physiol. 246, 549-569, 1975. 